\title{
Role of Focusing Distance in Picosecond Laser-Induced Cu Plasma Spectra
}

\author{
Linyu Chen $\mathbb{D}^{1},{ }^{1}$ Hu Deng, ${ }^{1}$ Zhixiang Wu, ${ }^{1}$ Zhonggang Xiong, ${ }^{1,2}$ Jin Guo, ${ }^{1,3}$ Quancheng Liu, ${ }^{1}$ \\ Akwasi Danso Samuel, ${ }^{1}$ and Liping Shang $\mathbb{D}^{1}$ \\ ${ }^{1}$ School of Information Engineering, Southwest University of Science and Technology, Mianyang 621010, China \\ ${ }^{2}$ School of Mechanical Engineering, Guilin University of Aerospace Technology, Guilin 541004, China \\ ${ }^{3}$ School of Intelligent Technology, Tianfu College of Southwestern University of Finance and Economics, Mianyang 621000, China
}

Correspondence should be addressed to Liping Shang; shangliping@swust.edu.cn

Received 31 August 2021; Revised 26 November 2021; Accepted 13 January 2022; Published 25 January 2022

Academic Editor: Anna Cimmino

Copyright (c) 2022 Linyu Chen et al. This is an open access article distributed under the Creative Commons Attribution License, which permits unrestricted use, distribution, and reproduction in any medium, provided the original work is properly cited. The publication of this article was funded by $\mathrm{SCOAP}^{3}$.

\begin{abstract}
To study the effects of focusing distance on the characteristics of copper plasma, a picosecond laser is utilized to ablate a pure copper plate to generate a plasma spectrum. Following numerous experiments on the subject, three significant factors are determined: lens focal length, pulse energy, and the lens-to-sample distance. These factors are employed to analyze the spectral intensity, plasma temperature, and electron density in the local thermodynamic equilibrium (LTE) and optically thin condition. Due to the shielding effects of mixed plasma, the strongest spectral intensity is achieved in the prefocused case, no matter how much beam irradiance is employed. The more intensive the beam irradiance is, the more the optimal position is distant from the focal point. The variation of plasma temperature and electron density showed a peak in the prefocused case, which is consistent with the trend of spectral intensity. For the case of extremely high irradiance (on the focus), the shielding effects become seriously, and the resultant above three factors decreased sharply. When a longer-focal-length lens is employed, the spectral intensity exhibited an obvious bimodal trend. In the prefocused case, a longer-focal-length lens is helpful to eliminate the effects of the roughness of the target surface compared with a shorter one. Finally, the assumed LTE is validated by McWhirter relation, plasma relaxation time, and diffusion length, and the optically thin condition was also validated by spectral intensity ratio. We hope that this work could be an important reference for the future design of highly optimized experiments for Calibration-Free Laser-Induced Breakdown Spectroscopy (CF-LIBS).
\end{abstract}

\section{Introduction}

Alongside the advancements in high-performance lasers and novel chemometrics methods in the last 30 years, laserinduced breakdown spectroscopy (LIBS) has become an attractive technique, with much potential, for the analysis of elements of materials [1]. LIBS offers distinct advantages for in situ, real-time, standoff, and contactless detection, and it requires less sample preparation and consumption [2]. This technique is widely applied to the fields of energetic materials [3], metallurgical engineering [4], space exploration [2], industry manufacture [5], food safety [6], environmental monitoring [7], agricultural product [1], resources and energy [8], archaeology [9], and clinical diagnostics
[10]. However, the stability and repeatability of the plasma emission, along with the detection accuracy, are easily altered by the wavelength of the input light, pulse duration and its energy, the distance of lens-to-sample, and the target characteristics.

To enhance the spectral intensity and improve the analytical precision, a larger number of insightful studies have been offered within the past ten years. For example, Fahad and Abrar used a pulse of wavelength $1064 \mathrm{~nm}$ and $532 \mathrm{~nm}$ to analyze the plasma characteristics of marble calcite [11]. When a femtosecond laser is used to ablate a copper target, the spectral intensity of $\mathrm{Cu}(\mathrm{I})$ and $\mathrm{Zn}(\mathrm{I})$ is higher when the laser is circularly polarized rather than linearly polarized [12]. The dependence of the spectral intensity of Si on the 
position of a lens, at different sample temperatures, is investigated by Zhang et al. [13], while Guo et al. analyzed the influence of the distance between the lens and the sample on spectral intensity and the vibrational temperature of the $\mathrm{CN}$ band, by using a nanosecond pulse to ablate polymethyl methacrylate [14]. Harilal et al. investigated the spot size effects on propagation dynamics [15] and conversion efficiency [16] of laser-produced plasma in vacuum and argon atmosphere; the results show that the sharpening of plasma plume depends heavily on the spot size. An improvement of up to three times has been achieved by Carvalho et al. in the measurement precision, when the particle size of plant pellets is less than $20 \mu \mathrm{m}$ and larger than $150 \mu \mathrm{m}$ [17]. By combining spatial confinement and double-pulse, the intensity of the $\mathrm{Cr}$ lines has a prominent enhancement factor of 168.6 [18]. Of course, there are other methods for enhancing spectral intensity, such as magnetic fields [19], sample heating [20], and nanoparticle coating [21].

The above work mainly uses a nanosecond or a femtosecond laser to generate a plasma spectrum. Compared with these two lasers, picosecond laser has appropriate pulse width with higher pulse energy, which can increase the ablation volume and spectral intensity (for femtosecond pulse), and may alleviate the effect of plasma shielding as well (for nanosecond pulse). It is well known that the pulse duration and beam irradiance of a laser are two crucial parameters in the process of laser-matter interaction. The longer the duration, the more heat effects on plasma or the surface of a target. When a femtosecond pulse illuminates the target, the phenomenon of target heating and vaporing will occur a few picoseconds after the laser pulse, and the target plasma-laser interaction never comes [22]. For nanosecond LIBS, the enhanced spectrum is attributed to plasma reheating and increased ablation mass. In the case of longer duration or enhanced beam irradiance, the effects of plasma shielding are a considerable factor in the procedure of plasma formation. Compared with femtosecond, the pulse energy of a picosecond laser is up to a few hundred $\mathrm{mJ}$, which can increase the spectral intensity caused by plasma reheating or ablation mass [23]. The mass removal from the target is more reproductive, and the intensity-tobackground ratio of a spectral line is higher when using a picosecond rather than a nanosecond laser [24].

Generally, plasma shielding is an undesirable phenomenon in LIBS application (especially in nanosecond LIBS). For femtosecond and nanosecond laser-induced plasma, the variation of line intensity and plasma temperature shows a similar trend with the ranged lens-to-sample distance, while the plasma formation and shielding effects are not completely identical. Due to huge differences in pulse duration, the characteristic of picosecond plasma may different from the femtosecond/nanosecond plasma. This work attempts to analyze the influence of focusing distance on the characteristics of picosecond laser-induced $\mathrm{Cu}$ plasma. A series of experiments are conducted in this work using a set of fixed focal lengths, pulse energies, and lens-tosample distances. Those are achieved by using three lenses of focal length, $f=50,100$, and $150 \mathrm{~mm}$. The line intensity and its optimal focusing distance are thoroughly investigated for pulse energy ranging from $12 \mathrm{~mJ}$ to $44 \mathrm{~mJ}$. Under the local thermodynamic equilibrium (LTE) and optically thin condition, we have observed the variation of plasma temperature and electron density with increased focusing distance.

\section{Materials and Methods}

A schematic for picosecond laser-induced plasma spectroscopy is shown in Figure 1. The copper plasma is produced with a Q-switched Nd: YAG laser (SL234, Ekspla) operating at an FWHM (full width at half maximum) duration of 120 ps. This picosecond laser outputs at a maximum energy of $250 \mathrm{~mJ}$ with a stability of $1.5 \%$ when working at its fundamental wavelength of $1064 \mathrm{~nm}$. The laser pulse is reflected by the mirror (denoted by M1 in Figure 1), and then, it is focused on the surface of a copper plate via a lens, L1. This lens is fixed on a vertical linear translation 1D-stage (NRT150, Thorlabs), while the copper plate is located on a $3 \mathrm{D}$-stage. On the right-hand side of Figure 1, the parameter $H$ represents the lens-to-sample distance; this can range from $40 \mathrm{~mm}$ to $160 \mathrm{~mm}$ by a movement of the $1 \mathrm{D}$-stage. To ensure the accurate $H$, the size of ablated spot produced by low pulse energy $(\sim 0.15 \mathrm{~mJ})$ on a piece of photographic paper is measured by an industrial microscope (LV100D, Nikon) and used to estimate the practical focal position of the lens with $f=50 \mathrm{~mm}$. The Cu plasma spectrum is collected via two plano-convex lenses, L2 and L3 (with focal lengths $150 \mathrm{~mm}$ and $100 \mathrm{~mm}$, respectively) and then coupled to a multichannel fiber spectrometer (AvaSpec-ULS2048-8USB2, Avantes). The wavelength of this spectrometer ranges from $200 \mathrm{~nm}$ to $1070 \mathrm{~nm}$, and the resolution is greater than $0.06 \mathrm{~nm}$ for $312 \mathrm{~nm}-564 \mathrm{~nm}$. The trigger and delay of the picosecond laser and spectrometer are controlled manually by the digital delay/pulse generator DG645, with a burst mode to generate an external $8 \mathrm{~Hz}$ trigger.

Before the experiment is begun, a calibration light source (AvaLight-CAL-Mini, Avantes) is used to calibrate the sensitivity of the spectrometer. According to the method reported in the literature [25], the instrumental broadening $(\sim 0.045 \mathrm{~nm})$ is estimated by a pair of $\mathrm{Hg}$ lines (576.96 and $579.06 \mathrm{~nm}$ ) emitted from this lamp. The pure copper plate is moved at a constant speed to ensure that each shot provides a fresh point. Each spectrum data is an average of 10 continuous shots. The interval between the laser pulse and the actual trigger of the spectrometer is set to $1.3 \mu \mathrm{s}$, and the gate width of the spectrometer exhibits a minimum value of $1.05 \mathrm{~ms}$. The whole experiment was carried out in air atmosphere, and air humidity ranges from $28 \%$ to $32 \%$ at room temperature.

\section{Results and Discussion}

3.1. Spectral Intensity at a Fixed Focal Length. At first, the pulse energy is set to $20 \mathrm{~mJ}$, and a lens with $f=150 \mathrm{~mm}$ is chosen to illuminate the surface of copper plate. When the focusing distance $(H)$ is $149 \mathrm{~mm}$ (prefocused case), the $\mathrm{Cu}$ plasma spectrum is given in Figure 2. In this circumstance, there are nine spectral lines with reasonably strong intensities. By referring to the NIST database and related report 


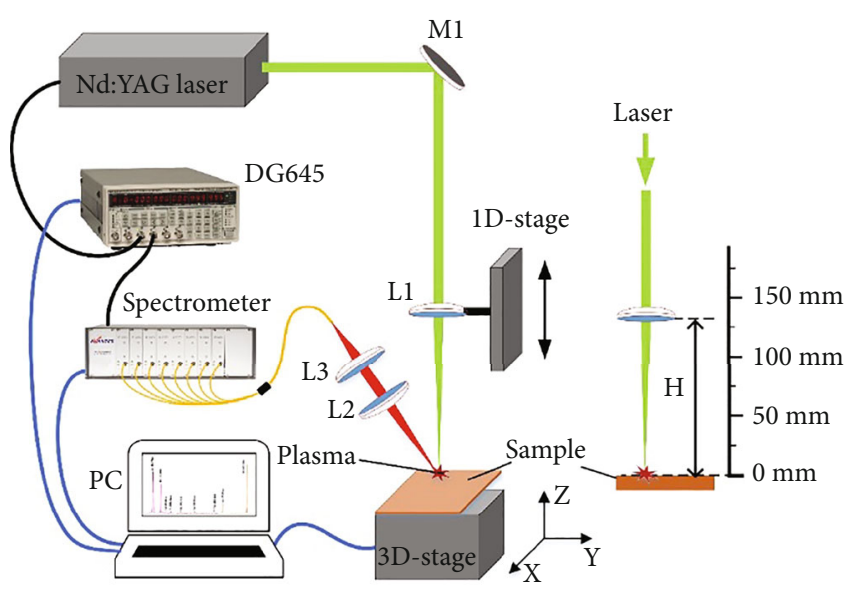

Figure 1: Schematic for picosecond laser-induced plasma spectroscopy with a variable lens-to-sample distance.

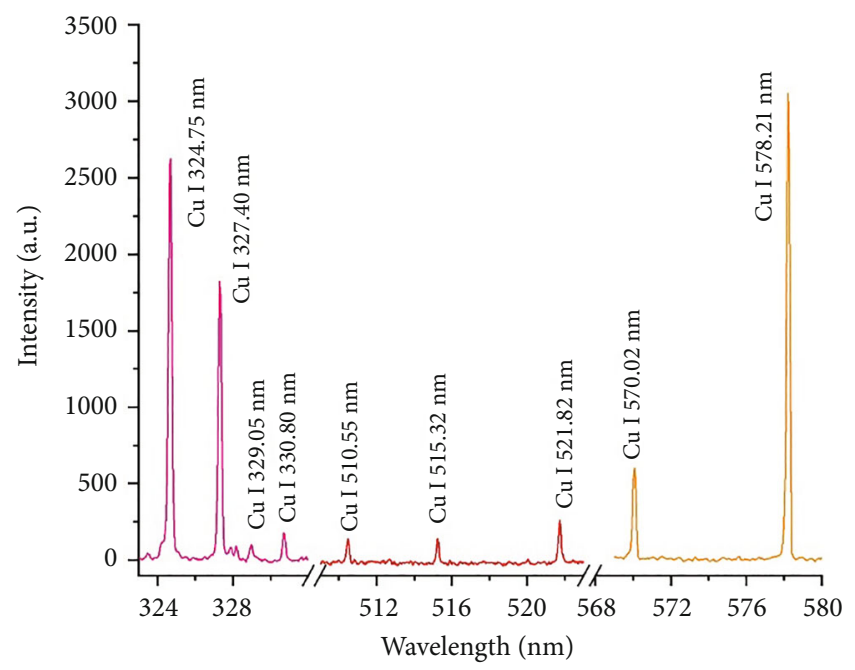

Figure 2: Graph showing the plasma spectra for a $\mathrm{Cu}$ plate at $H$ $=149 \mathrm{~mm}(f=150 \mathrm{~mm}$, pulse energy is $20 \mathrm{~mJ})$.

[26], those spectral lines clearly all relate to the $\mathrm{Cu}$ atomic lines at wavelengths of $324.75,327.40,329.05,330.80$, $510.55,515.32,521.82,570.02$, and $578.21 \mathrm{~nm}$.

When $H$ ranged from $144.6 \mathrm{~mm}$ to $156 \mathrm{~mm}$, the line intensity of the three strongest lines, relating to $\mathrm{Cu} \mathrm{I}$ at $324.75,327.40$, and $578.21 \mathrm{~nm}$, is shown in Figure 3. We can determine from this graph that, in comparison to the continuous spectral intensity of the background, these three spectral lines are much larger and easily distinguishable from the rest. Two intensity peaks arise in the prefocused $(H=149 \mathrm{~mm})$ and defocused $(H=151 \mathrm{~mm})$ cases, but the prefocused one is more intensive than the defocused one. For the prefocused case $(H<150 \mathrm{~mm})$, as the $H$ increased, the coupling efficiency between the laser pulse and the sample is increased firstly due to the reduced spot size, resulting in a stronger spectrum and an opaque plasma with higher density. Once the plasma density reaches a critical value, a thin layer forming above the target surface will absorb the subsequent energy and prevent the laser from propagating

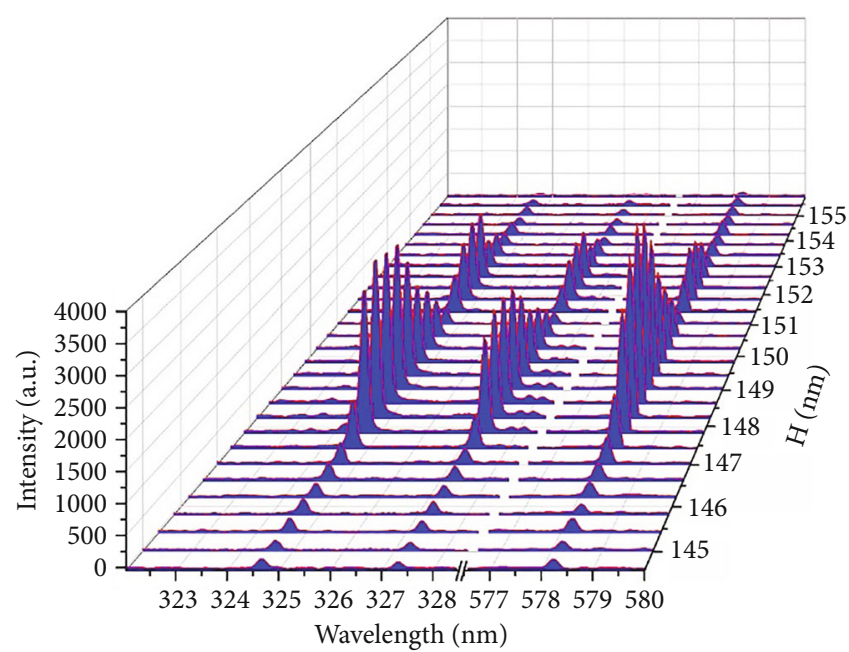

FIgURE 3: Graph showing the spectra of the three $\mathrm{Cu}(\mathrm{I})$ lines with $H(f=150 \mathrm{~mm}$, pulse energy is $20 \mathrm{~mJ})$.

to the target, which is the phenomenon of plasma shielding [26]. Referring to the related report on laser-material interaction [27], the pulse duration of $120 \mathrm{ps}$ is sufficient to maintain the target plasma encounters the rest incident energy. When the focus point is located on the target surface ( $H=150 \mathrm{~mm}$, i.e., on the focus), the plasma shielding dominates the energy, and the spectral intensity drops sharply. The results indicated that the ablated copper surface is modified by both the beam irradiance of the pulse and the effects of plasma shielding.

3.2. Spectral Intensity at Different Focal Lengths. To achieve the optimal lens-to-sample distance (with high line intensity) and analyze the effects of plasma shielding on line intensity at different focal lengths, the pulse energy is set to $20 \mathrm{~mJ}$, and the lens L1 now has either $f=50 \mathrm{~mm}$ or $100 \mathrm{~mm}$. Figure 4 shows the variation of atomic line intensity of $\mathrm{Cu}$ at $324.75,327.40,510.55,515.32,521.82,570.02$, and $578.21 \mathrm{~nm}$.

Examining the system using a lens with $f=150 \mathrm{~mm}$, when $H$ changes from $144.5 \mathrm{~mm}$ to $149 \mathrm{~mm}$ (at the prefocused case), the intensity of the seven spectral lines of Figure 4(a) initially increases more and more rapidly. This is because the increasing $H$ reduces the spot size on the target surface, causing a nonlinear increase in the beam irradiance. In the case of low beam irradiance, as long as the electron density is above the critical threshold, an increased beam irradiance will lead to more energy absorption in the region of laser-supported detonation wave (LSDW) [28], increasing the ablated volume of the copper surface and plasma emission. The maximum spectral intensity occurs at $H=148.6 \mathrm{~mm}$, which is about 2.3 times the intensity of the focal point $(H=150 \mathrm{~mm})$. While using the lens with $f$ $=100 \mathrm{~mm}$, the highest intensity can be obtained at $H=$ $99.2 \mathrm{~mm}$, and its spectral intensity is about 2.6 times that of the focal point.

When $H$ is within reach of $150 \pm 1 \mathrm{~mm}$ (approximately on the focus), during the copper plate moving from within the focal point (prefocused case) to outside it (defocused case), 


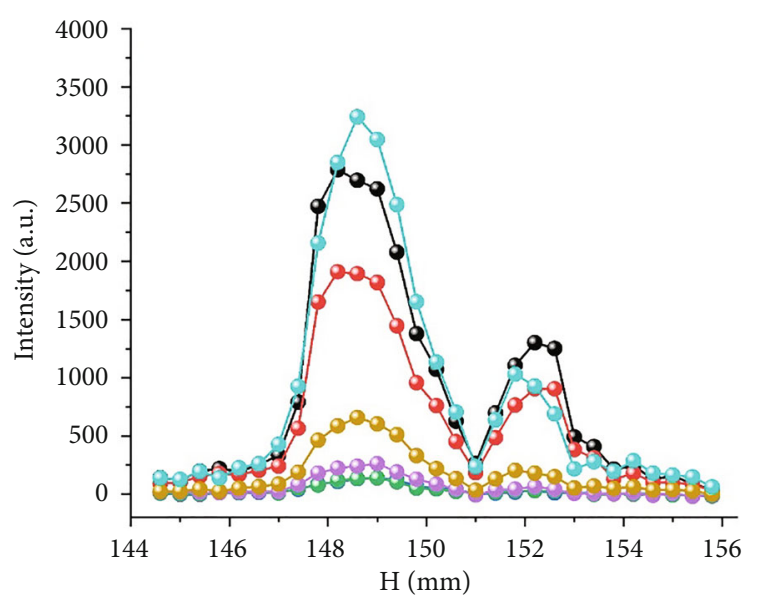

(a)

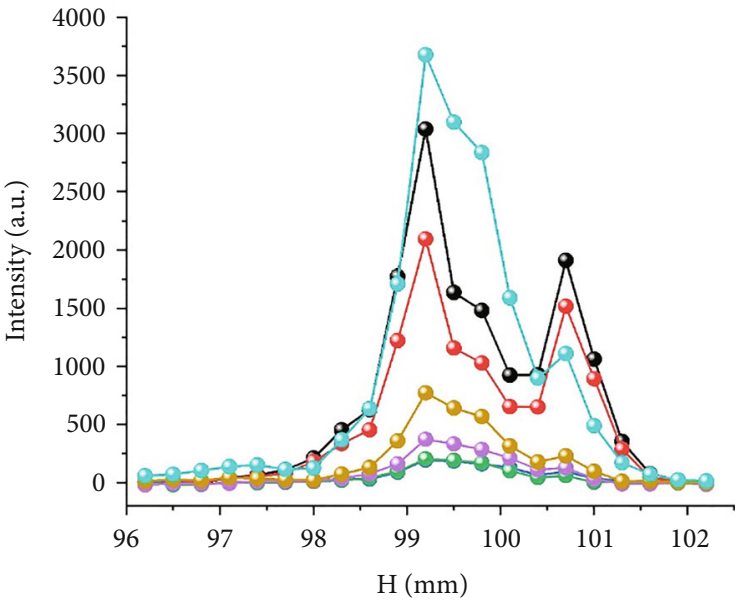

(b)

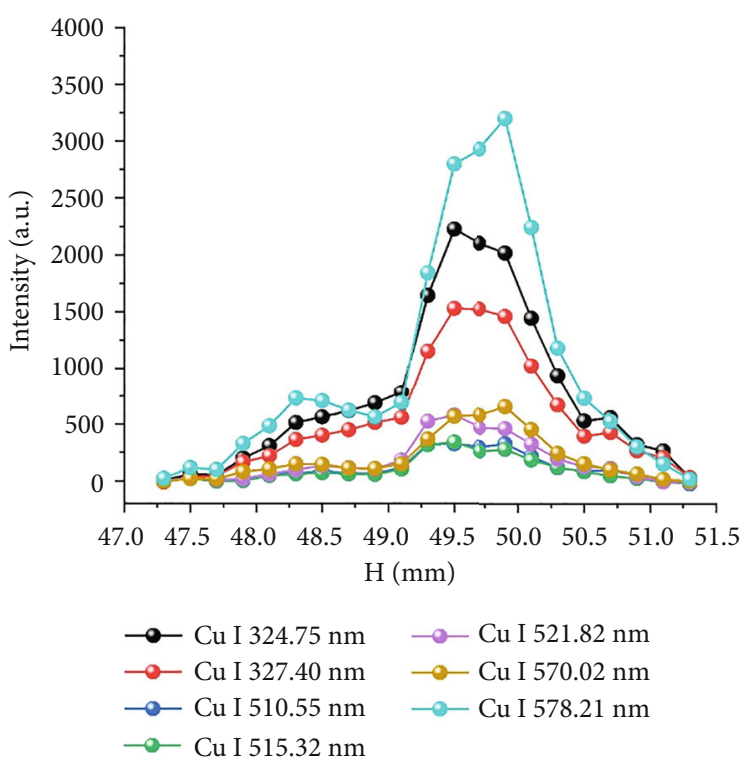

(c)

FIGURE 4: Graphs showing that the intensities of $\mathrm{Cu}$ atomic lines (i.e., $\mathrm{Cu}(\mathrm{I})$ at $324.75 \mathrm{~nm}, 327.40 \mathrm{~nm}, 510.55 \mathrm{~nm}, 515.32 \mathrm{~nm}, 521.82 \mathrm{~nm}$, $570.02 \mathrm{~nm}$, and $578.21 \mathrm{~nm}$ ) change with $H$, when using the lens L1 with a focus length of (a) $150 \mathrm{~mm}$, (b) $100 \mathrm{~mm}$, and (c) $50 \mathrm{~mm}$. The pulse energy is $20 \mathrm{~mJ}$.

the line intensity shows a similar sharp downward trend and reaches the minimum value at $H=151 \mathrm{~mm}$. When the copper plate is near the focal point, the laser pulse has an extremely high irradiance. The plasma plume above the copper surface becomes opaque to the laser pulse [29], which produces a strong shielding effect and results in almost no energy transferring to the surface of copper. It greatly reduces the ablation mass on the copper surface and ultimately leads to a weak spectral intensity.

For the defocus case (typically $H>150 \mathrm{~mm}$ ), another peak intensity point is reached at $H=152 \mathrm{~mm}$. This is because the decreased beam irradiance produces a low electron density, which strengthens the coupling efficiency between the mixed plasma and the target, resulting in the above three parameters increasing again [28]. In this work, the high-fluence laser transmits through the air atmosphere; the self-focusing effect might occur and help to relieve the defocusing effect of the lens and enhance the irradiate fluence on the target surface. When $H>152 \mathrm{~mm}$, the increased size of the focusing spot leads to a declined beam irradiance, which gradually reduces the ablation mass and the spectral intensity until the beam irradiance is below the breakdown threshold of copper.

Following the examination of the three lenses with different focal lengths, we determined that when $f=100 \mathrm{~mm}$ or $150 \mathrm{~mm}$, the spectral intensities show an obvious bimodal trend with increasing $H$, while this trend does not occur for $f=50 \mathrm{~mm}$. The width of the first peak indicated that when the lens is prefocused, a longer-focal-length lens is helpful to eliminate the effects of the roughness of the target surface on line intensity compared with a shorter-focal-length lens [30]. Meanwhile, the distinct plasma shielding is easily observed mainly due to the air breakdown above the target surface. Besides, the ranged spot size on the target surface 
is another essential factor for inducing plasma shielding. In the air atmosphere, the plasma plume structure is long and narrow when using a long-focal lens but relatively flat when a short length is employed. The geometric size of the plasma plume impacts the coupling efficiency between the laser pulse and the target surface [16], which directly alters the ablation mass and spectral intensity.

3.3. Plasma Emission Dependence on Pulse Energy. It is known that the beam irradiance directly affects the ablation mass of the target surface, which in turn alters the coupling efficiency between the target and the incident beam, and finally induce the changed spectral intensity of the plasma. The intensity change of line $578.21 \mathrm{~nm}$ is selected as the one used to analyze in-depth the effects of the pulse energy. Taking the lens with $f=150 \mathrm{~mm}$ as an example, with the pulse energy ranging from $12 \mathrm{~mJ}$ to $44 \mathrm{~mJ}$, the line intensity at $578.21 \mathrm{~nm}$ with increased $H$ is shown in Figure 5. It is observed that the spectral intensity and its maximum spectral intensity are enhanced with the increased pulse energy. The maximum spectral intensity and its corresponding $H$ values indicate that the best location of the focusing lens is determined in the prefocused case, rather than on the focal point with the highest energy fluence. The higher the pulse energy is, the farther the target (the maximum spectral intensity) from the focal point is.

With pulse energy ranging from $12 \mathrm{~mJ}$ to $44 \mathrm{~mJ}$, the corresponding maximum intensity of $578.21 \mathrm{~nm}$ is shown in Figure 6. After optimizing the lens-to-sample distance, the spectral intensity shows a linear increasing trend with the increased pulse energy, no matter which lens is employed. When the pulse energy is exceeded $20 \mathrm{~mJ}$, and a lens with $f=100 \mathrm{~mm}$ is used, the maximum spectral intensity is more sensitive to a change in the pulse energy.

With increasing pulse energy, the optimal lens-tosample distances (with the maximum spectral intensity) are calculated and presented in Figure 7. We found that, when the same lens is used to both focus and ablate the copper surface, the larger the pulse energy the closer the lens is to the target with maximum spectral intensity. Taking the lens with a focal length of $150 \mathrm{~mm}$ as an example, when the pulse energy is $12,20,28,36$, and $44 \mathrm{~mJ}$, comparing the positions of the focal point, the lens moves downwards by $0.6,1.4,2,2.4$, and $3.5 \mathrm{~mm}$, respectively. Under the same pulse energy, the maximum spectral intensity is obtained when the distance of the target surface to the focus is greater (if a longer focal length is used). Taking pulse energy of $36 \mathrm{~mJ}$ as an example, when lenses with $f=50,100$, and $f=150 \mathrm{~mm}$ are used, relative to each focal point, the copper plate moves forward by $0.6,1.5$, and $2.4 \mathrm{~mm}$, respectively.

Once the pulse energy and lens are fixed, it is well known that the farther the sample is from the focal position, the larger the spot size and the lower the beam irradiance, with the highest beam irradiance at the focal point. However, the spectral intensity at the focal point is not the strongest (as shown in Figure 4), and it is much lower than that of the prefocused case. Therefore, we can conclude that the higher the beam irradiance, the more serious the effects of

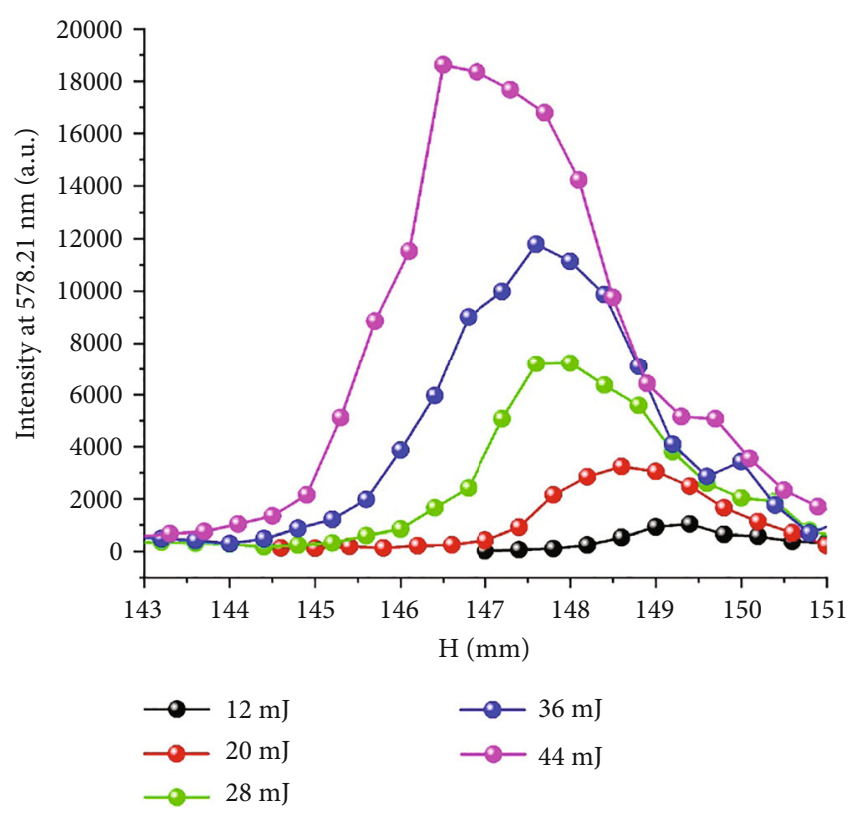

FIgURE 5: Evolution of spectral intensity with $H$ at different pulse energies when $f=150 \mathrm{~mm}$.

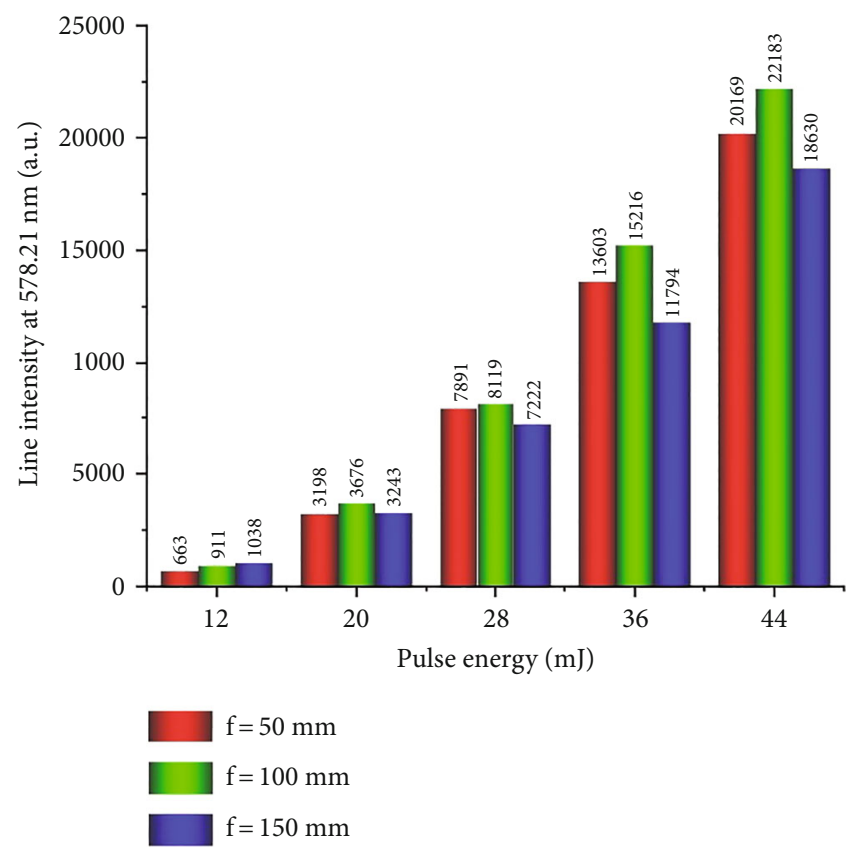

FIgURE 6: Maximum spectral intensity of $\mathrm{Cu}(\mathrm{I})$ at $578.21 \mathrm{~nm}$ for various pulse energies.

plasma shielding. It is speculated that the increased rate of plasma shielding is lower than that of pulse energy, though the corresponding effects of plasma shielding are enhanced. Finally, the higher laser energy deposits on the copper surface and leads to an enhanced ablation mass and plasma emission. While the optimal copper position (with maximum spectral intensity) will be closer to the lens (as shown in Figure 7) and the size of the ablated spot will become larger. Besides, the absorbed energy will heat the plasma and contribute to the emission intensity. 


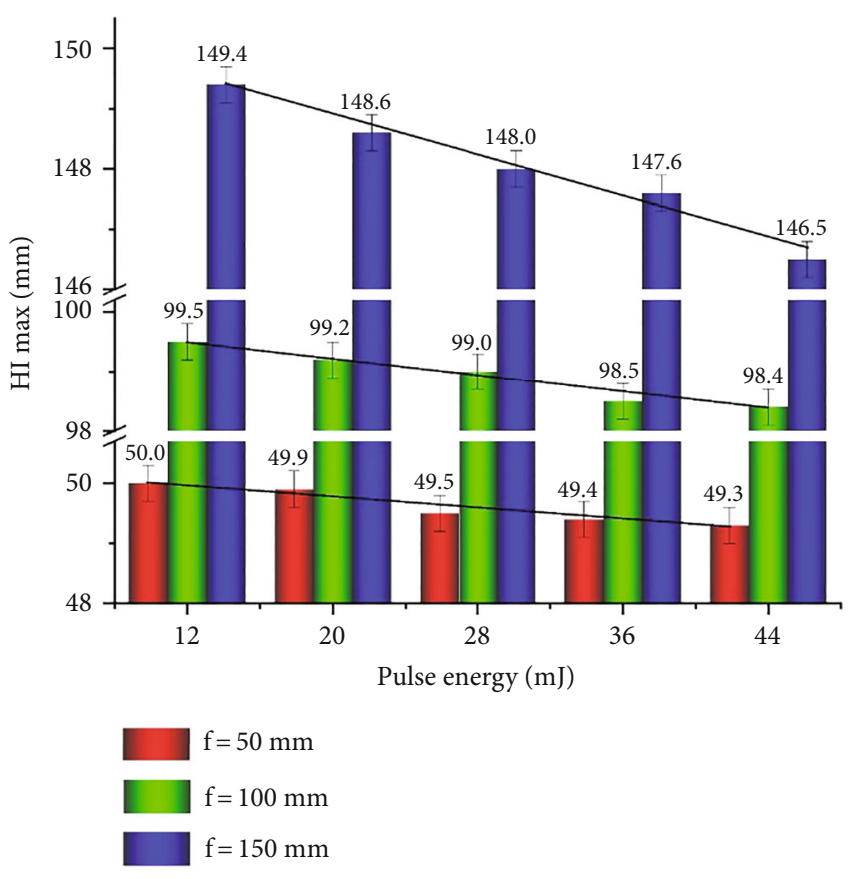

FIGURE 7: Graph showing the lens-to-sample distance when the maximum spectral intensity varies with pulse energy (HImax represents the lens-to-sample distance with maximum spectral intensity. The error bar is $0.3 \mathrm{~mm}$, coming from the procedure of $H$ calibration.).

In the course of plasma shielding, the absorption energy in plasma is tightly related to the combined effects of the ablation spot size, beam irradiance, plasma geometry, and plasma shielding [29]. Any severe shielding weakens the coupling efficiency between the laser pulse and the target surface and reduces the ablation mass on the target surface, finally resulting in a weaker emission spectrum intensity.

3.4. Plasma Temperature and Plasma Density. The existence of plasma shielding induces the plasma to absorb the rest of pulse energy. Besides the emitted spectrum, the thermodynamic parameter, the plasma temperature, and electron density will be affected by the changed ablation or reheating energy. In practical applications of LIBS, the local thermodynamic equilibrium (LTE) and optically thin are generally assumed to evaluate the plasma characteristics. When the above assumptions are established, the self-absorption effects in the procedure of plasma emission can be ignored [31]. The plasma temperature $T(\mathrm{~K})$ is determined from the fitted slope of the Boltzmann plot.

$$
\ln \frac{I_{1} \lambda_{1}}{A_{1} g_{1}}-\ln \frac{I_{2} \lambda_{2}}{A_{2} g_{2}}=-\frac{1}{K_{b} T}\left(E_{1}-E_{2}\right),
$$

where $\lambda_{1}$ and $\lambda_{2}$ represent the central wavelength of two atomic spectral lines with their corresponding spectral intensity being $I_{1}$ and $I_{2}, A_{1}$ and $A_{2}$ represent the spontaneous transition probabilities of spectral lines $\lambda_{1}$ and $\lambda_{2}$ from high energy levels to low energy levels, $g_{1}$ and $g_{2}$ are the degeneracy of the upper levels of $E_{1}$ and $E_{2}$, respectively, and $K_{b}$ is the Boltzmann constant $(\mathrm{eV} / \mathrm{K})$.
Three $\mathrm{Cu}$ atomic lines at 510.55, 515.32, and $521.82 \mathrm{~nm}$ are used to determine the precise plasma temperature. The above lines not only match the following four physical features, such as a nonresonance line with less self-absorption, higher transition probability, individual line without interference and deformation, and enough difference of the upper-level energy of two lines but also within the same channel with identical coupling efficiency between the plasma and the optical fiber. The spectroscopic parameters of the above three $\mathrm{Cu}$ atomic lines can be obtained in the NIST database.

Generally, Stark broadening of the spectral linewidth is mainly caused by the collisions of electrons and ions. Both the linear and the quadratic Stark effect are encountered in spectroscopy. Only the hydrogen atom and $\mathrm{H}$-like ions exhibit the linear Stark effect, whereas all other atoms exhibit the quadratic Stark effect. When calculating the electron density, the FWHM of $H_{\alpha}$ line profile can be calculated easily with greater accuracy than $\mathrm{Cu}$ atomic lines. Stark broadening was assumed to fit a Lorentz line shape. The electron density $N_{e}$, a function of FWHM $\Delta \lambda$, is determined by the following expression [32].

$$
N_{e}=8.02 \times 10^{12}\left(\frac{\Delta \lambda}{\alpha}\right)^{3 / 3} \mathrm{~cm}^{-3}
$$

where $\lambda$ is the FWHM of the line in $\AA$ and $\alpha$ is the reduced Stark profiles in $\AA$ as tabulated by Kepple (i.e., $\alpha$ is 0.0149 , line 2 in Table 1, page 322.) [33].

Here, taking the lens with a focal length of $150 \mathrm{~mm}$ as an example, when pulse energy is $44 \mathrm{~mJ}$, the variation of plasma temperature and electron density with increased $H$ is shown in Figure 8. It is found that the peak is located around $H$ $=146.5 \mathrm{~mm}$, which is corresponding to the first peak of spectral intensity in Figure 5.

When $H$ ranged from $143 \mathrm{~mm}$ to $146.5 \mathrm{~mm}$, the copper surface absorbed the pulse energy and induce the increased temperature of the electrons and lattices. If the electrons absorb enough incident photons, it will emit along the opposite direction of the incident beam with high speed and high density and break down the free air and produce the initial weak air plasma (weak spectral line $H \alpha$ was observed) above the target surface [27]. Once the beam irradiance or electron density reaches a certain critical threshold, the shielding effects will occur in the mixed plasma consisting of target plasma and air plasma. Due to the shielding effects, the mixed plasma absorbs a part of the pulse energy through the electron-ion inverse-bremsstrahlung or the electronneutral inverse-bremsstrahlung processes [31]. The spectral intensity, plasma temperature, and electron density began to show a peak value, which means that the plasma shielding is relatively weak. When $H>146.5 \mathrm{~mm}$, the enhanced beam irradiance will aggravate the shielding effects. It implies that less and less energy can reach the target surface, and that mixed plasma will absorb more energy to strengthen the procedure of ionization and collision between the different particles. Although the increased energy is converted into the internal thermal energy of the mixed plasma through 


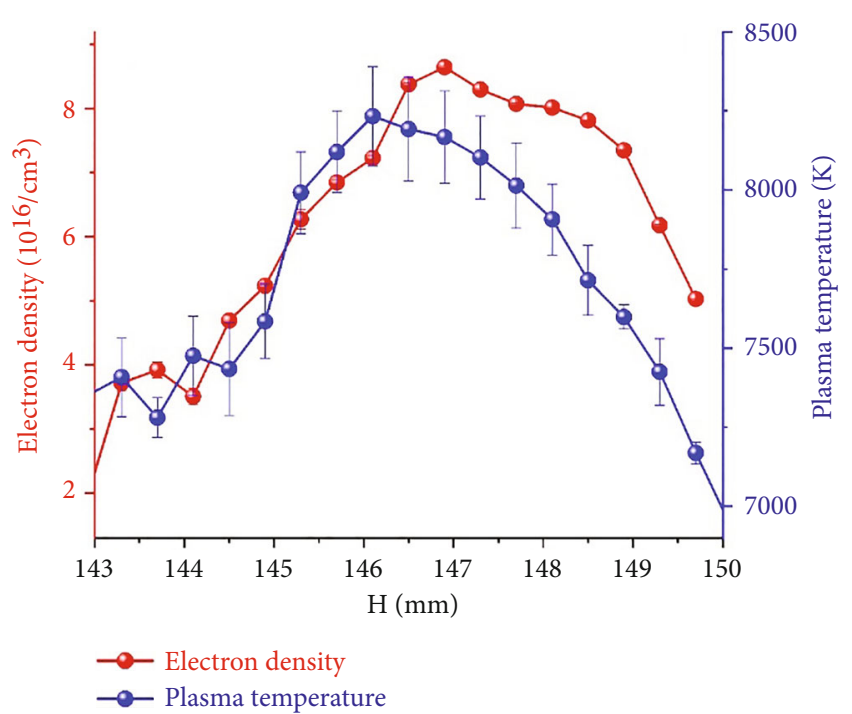

FIGURE 8: Evolution of electron density and plasma temperature at different $H$.

inverse-bremsstrahlung radiation, the plasma temperature has shown a declined slope. We deem that the narrow geometry of mixed plasma aroused by the smaller size of ablation spot alleviate the reheating effects on plasma. When the target is above the focal point (prefocused case), the variation of plasma temperature suggested that the rise in plasma temperature is governed by the ablation mass, rather than the plasma reheating.

3.5. Validity of Assumption. LTE refers to the existence of thermal equilibrium within a small region of the plasma. In LTE plasma, the collisional processes of excitation and de-excitation dominate the radiative processes, which require the electron density to be following the McWhirter relation [34].

$$
N_{e} \geq 1.6 \times 10^{12} T^{1 / 2}(\Delta E)^{3},
$$

where $\Delta E$ represents the difference between the upper and lower energy $(\mathrm{eV})$ levels of the spectral line. According to the physical parameters mentioned in the last section, the minimum electron density can be approximately calculated as $0.202 * 10^{16} / \mathrm{cm}^{3}$ when the plasma temperature is up to $8233 \mathrm{~K}$. It is lower than the experimentally obtained values at any $H$ in Figure 8; the McWhirter criterion is satisfied.

McWhirter criterion is a necessary but sufficient condition for the LTE in the homogeneous and stationary plasma. For nonstationary and inhomogeneous plasma, two additional criteria, plasma relaxation time and diffusion length, impose limitations regarding the temporal and spatial gradients of thermodynamic parameters ( $T$ and $N_{e}$ ). In a homogeneous and transient plasma, the time required to establish excitation and ionization equilibrium (i.e., relaxation time) should be much shorter than the time of variation in $T$ and $N_{e}$. This criterion is given by the following inequality [34].

$$
\begin{gathered}
\frac{T\left(t+\tau_{\text {rel }}\right)-T(t)}{T(t)} \\
\frac{N_{e}\left(t+\tau_{\text {rel }}\right)-N_{e}(t)}{N_{e}(t)} \ll 1,
\end{gathered}
$$

where the $\tau_{\text {rel }}$ is the plasma relaxation time, which can be expressed as [34].

$$
t_{\text {rel }}=\frac{6.3 \times 10^{4}}{N_{e} f_{m n}\langle g\rangle} \Delta E_{n m}\left(K_{b} T\right)^{1 / 2} \exp \left(\frac{\Delta E_{n m}}{K_{b} T}\right),
$$

where $f_{m n}$ is the oscillator strength of the $\mathrm{m}-\mathrm{n}$ transition (ranging from 0.42 to 0.54 , Table 1.1 in literature [35]). $\langle g\rangle$ is the effective Gaunt factor (dimensionless, 0.04, Figure 1 in literature [34]). $\triangle E_{n m}$ is the energy difference between the upper and lower level. For line $521.82 \mathrm{~nm}$, the relaxation time $\tau_{\text {rel }}$ is estimated at $\sim 3.3$ nanoseconds, which is much shorter than the observation time of the spectrometer in this work. It means that the LTE is satisfied in the homogenous and transient plasma.

To consider the valid LTE as overall as possible, diffusion length $\Delta x$ should be estimated in the inhomogeneous and transient plasmas. It requires that the diffusion length of species, during a period of the order of the relaxation time $\left(\tau_{\text {rel }}\right)$ to the equilibrium, is shorter than the length of variation in temperature and electron number density. The condition for LTE at the position $x$ can be expressed as [34].

$$
\begin{aligned}
\frac{T(x+\Delta x)-T(x)}{T(x)} & \ll 1, \\
\frac{N_{e}(x+\Delta x)-N_{e}(x)}{N_{e}(x)} & \ll 1,
\end{aligned}
$$

the diffusion length $\Delta x$ is expressed as [34].

$$
\Delta x \approx \frac{1.4 \times 10^{12}}{N_{e}}\left(k T_{e}\right)^{3 / 4}\left(\frac{\Delta E_{n m}}{M_{A} f_{m n}\langle g\rangle}\right)^{1 / 2} \exp \left(\frac{\Delta E_{n m}}{2 k T_{e}}\right),
$$

where the $M_{A}$ is the relative mass of the species considered. Generally, the diffusion length must be at least one order of magnitude shorter than the plasma dimension. For $\mathrm{Cu}$ element, the value of $M_{A}$ is 29 . The diffusion length $\Delta x$ is estimated as $\sim 1.92 \mu \mathrm{m}$, while the plasma diameter can be estimated at $\sim 1 \mathrm{~mm}$. It means that the LTE is validated when the plasma is inhomogeneous and transient in this work.

As assumed in the calculating procedure of plasma temperature and electron density, the spectral lines are free from the self-absorption in optically thin plasma. For a plasma spectrum under the optically thin condition, the intensity relationship between two atomic lines must submit Equation (1). Especially for the lines originating from the same upper level in a specific element with the identical ionization stage, the theoretical doublet intensity ratio can be expressed by 
simplification of Equation (1) as follows [36].

$$
\frac{I_{1}}{I_{2}}=\frac{A_{1} g_{1} \lambda_{2}}{A_{2} g_{2} \lambda_{1}}
$$

where the three factors are the atomic inherent parameters of the transitions, which is independent of the experimental parameters.

Among the three $\mathrm{Cu}$ atomic lines used to calculate the plasma temperature, the double lines $515.32 \mathrm{~nm}$ and $521.82 \mathrm{~nm}$ have the same upper level. We can calculate the experimental intensity ratio of $521.82 \mathrm{~nm}$ to $515.32 \mathrm{~nm}$ $\left(I_{521.82 \mathrm{~nm}} / I_{515.32 \mathrm{~nm}}\right)$ with various $H$. The experimental value of $I_{521.82 \mathrm{~nm}} / I_{515.32 \mathrm{~nm}}$ ranges from 1.68 to 1.81 , which is close to the theoretical value of 1.85 obtained from Equation (6). Therefore, the observed plasma can exist in an optically thin condition. In conclusion, the assumption of LTE and optically thin condition is validated by minimum electron density, slower evolution, and theoretically spectral intensity ratio.

\section{Conclusion}

This work observed the spectral intensity characteristics of copper plasma using a ps LIBS under various conditions, such as different lens focal lengths, pulse energies, and focusing distances. The above phenomenon indicated that, with increased lens-to-copper surface distance, the difference of the focal length and pulse energy leads to the variation of beam irradiance under various behaviours. Both the beam irradiance and size of the ablation spot changed the geometric structure of the plasma and the particle density inside the plasma. The combined effects of these critical factors resulted in a variation of the coupling efficiency between the laser pulse and sample surface, which also affects the spectral intensity, plasma temperature, and electron density. When the pulse duration exceeds $5 \mathrm{ps}$, the pulse will be interacting with plasma and result in plasma reheating. The source of plasma may be different; a shorter picosecond laser will produce air plasma above the surface of a target, while the nanosecond laser will generally produce vapour-plasma from the removal mass [23]. Due to the effects of plasma shielding, the variation trend of spectral intensity with the focusing distance is consistent with the results of $8 \mathrm{~ns}$ and $50 \mathrm{fs}$ duration in literature [29,37], respectively. In this work, we deem that the main reason for the hump in Figure 4 is similar to that of nanosecond pulse, namely, the plasma reheating between the laser and mixed plasma, rather than the effects of self-focusing, defocusing, and refocusing in the air ionization by a femtosecond laser. As for the second peak in the defocused case, the selffocusing effects may help to enhance the coupling efficiency between the laser pulse and the sample. Finally, three criteria, the McWhirter relation, plasma relaxation time, and diffusion length, are used to validate the assumed LTE. And the theoretically spectral intensity ratio was employed to validate the optically thin condition. The present results provide a further understanding of the influence of beam irradiance on spectral intensity in picosecond LIBS. We hoped that this study could be a simple and effective method to improve detecting sensitivity without extraoptical elements in the next work.

\section{Data Availability}

According to the management agreement of our laboratory, the data used to support the findings of this study are available from the corresponding author upon reasonable request.

\section{Conflicts of Interest}

The authors declare no conflicts of interest.

\section{Acknowledgments}

This work was supported by the National Natural Science Foundation of China (Grant No. 11872058), the Sichuan Science and Technology Program of China (Grant Nos. 2019110 and 2019YFG0114), the Guangxi Natural Science Foundation of China (Grant No. 2019GXNSFBA185013), and the National Defense Basic Scientific Research Program of China (Grant Nos. JCKY2018404C007, JSZL2017404A001, and JSZL2018204C002).

\section{References}

[1] J. Peng, Y. He, J. Jiang, Z. Zhao, F. Zhou, and F. Liu, "Highaccuracy and fast determination of chromium content in rice leaves based on collinear dual-pulse laser-induced breakdown spectroscopy and chemometric methods," Food Chemistry, vol. 295, pp. 327-333, 2019.

[2] N. L. Lanza, S. M. Clegg, R. C. Wiens, R. E. McInroy, H. E. Newsom, and M. D. Deans, "Examining natural rock varnish and weathering rinds with laser-induced breakdown spectroscopy for application to ChemCam on Mars," Applied Optics, vol. 51, no. 7, pp. B74-B82, 2012.

[3] I. Gaona, J. Serrano, J. Moros, and J. J. Laserna, "Range-adaptive standoff recognition of explosive fingerprints on solid surfaces using a supervised learning method and laser-induced breakdown spectroscopy," Analytical Chemistry, vol. 86, no. 10, pp. 5045-5052, 2014.

[4] C. Pan, X. Du, N. An, Q. Zeng, S. Wang, and Q. Wang, "Quantitative analysis of carbon steel with multi-line internal standard calibration method using laser-induced breakdown spectroscopy," Applied Spectroscopy, vol. 70, no. 4, pp. 702$708,2016$.

[5] N. Zhao, D. Lei, X. Li et al., "Experimental investigation of laser-induced breakdown spectroscopy assisted with laserinduced fluorescence for trace aluminum detection in steatite ceramics," Applied Optics, vol. 58, no. 8, pp. 1895-1899, 2019.

[6] B. A. Alfarraj, H. K. Sanghapi, C. R. Bhatt, F. Y. Yueh, and J. P. Singh, "Qualitative analysis of dairy and powder milk using laser-induced breakdown spectroscopy (LIBS)," Applied Spectroscopy, vol. 72, no. 1, pp. 89-101, 2018.

[7] Y. Qu, Q. Zhang, W. Yin, Y. Hu, and Y. Liu, "Real-time in situ detection of the local air pollution with laser-induced breakdown spectroscopy," Optics Express, vol. 27, no. 12, pp. A790-A799, 2019.

[8] M. Burger, P. J. Skrodzki, L. A. Finney, J. Nees, and I. Jovanovic, "Remote detection of uranium using selffocusing intense femtosecond laser pulses," Remote Sensing, vol. 12 , no. 8 , p. $1281,2020$. 
[9] R. Gaudiuso, M. Dell'Aglio, O. De Pascale, S. Loperfido, A. Mangone, and A. De Giacomo, "Laser-induced breakdown spectroscopy of archaeological findings with calibration-free inverse method: comparison with classical laser-induced breakdown spectroscopy and conventional techniques," Analytica Chimica Acta, vol. 813, pp. 15-24, 2014.

[10] Q. Wang, G. Teng, X. Qiao et al., "Importance evaluation of spectral lines in laser-induced breakdown spectroscopy for classification of pathogenic bacteria," Biomedical Optics Express, vol. 9, no. 11, pp. 5837-5850, 2018.

[11] M. Fahad and M. Abrar, "Laser-induced breakdown spectroscopic studies of calcite ( $\mathrm{CaCO} 3)$ marble using the fundamental $(1064 \mathrm{~nm})$ and second $(532 \mathrm{~nm})$ harmonic of a Nd: YAG laser," Laser Physics, vol. 28, no. 8, p. 085701, 2018.

[12] Q. Wang, A. Chen, W. Xu, S. Li, Y. Jiang, and M. Jin, "Signal improvement using circular polarization for focused femtosecond laser-induced breakdown spectroscopy," Journal of Analytical Atomic Spectrometry, vol. 34, no. 6, pp. 1242-1246, 2019.

[13] D. ZHANG, A. CHEN, Q. WANG et al., "Effect of lens focusing distance on laser-induced silicon plasmas at different sample temperatures," Plasma Science and Technology, vol. 21, no. 3, p. 034009, 2019.

[14] K. Guo, A. Chen, and X. Gao, "Influence of distance between target surface and focal point on $\mathrm{CN}$ emission of nanosecond laser-induced PMMA plasma in air," Optik, vol. 208, article 164067, 2020.

[15] S. S. Harilal, "Influence of spot size on propagation dynamics of laser-produced tin plasma," Journal of Applied Physics, vol. 102, no. 12, article 123306, 2007.

[16] S. S. Harilal, R. W. Coons, P. Hough, and A. Hassanein, "Influence of spot size on extreme ultraviolet efficiency of laserproduced Sn plasmas," Applied Physics Letters, vol. 95, no. 22, article 221501, 2009.

[17] G. G. A. de Carvalho, D. Santos, L. C. Nunes, M. da Silva Gomes, F. de Oliveira Leme, and F. J. Krug, "Effects of laser focusing and fluence on the analysis of pellets of plant materials by laser-induced breakdown spectroscopy," Spectrochimica Acta Part B: Atomic Spectroscopy, vol. 74-75, pp. 162$168,2012$.

[18] L. B. Guo, B. Y. Zhang, X. N. He et al., "Optimally enhanced optical emission in laser-induced breakdown spectroscopy by combining spatial confinement and dual-pulse irradiation," Optics Express, vol. 20, no. 2, pp. 1436-1443, 2012.

[19] P. Liu, R. Hai, D. Wu, Q. Xiao, L. Sun, and H. Ding, “The enhanced effect of optical emission from laser induced breakdown spectroscopy of an Al-Li alloy in the presence of magnetic field confinement," Plasma Science and Technology, vol. 17, no. 8, pp. 687-692, 2015.

[20] Y. Wang, A. Chen, Q. Wang et al., "Influence of distance between focusing lens and target surface on laser-induced $\mathrm{Cu}$ plasma temperature," Physics of Plasmas, vol. 25, no. 3, p. 033302, 2018.

[21] X. Wen, Q. Lin, G. Niu, Q. Shi, and Y. Duan, "Emission enhancement of laser-induced breakdown spectroscopy for aqueous sample analysis based on $\mathrm{Au}$ nanoparticles and solid-phase substrate," Applied Optics, vol. 55, no. 24, pp. 6706-6712, 2016.

[22] A. Valenzuela, C. Munson, A. Porwitzky, M. Weidman, and M. Richardson, "Comparison between geometrically focused pulses versus filaments in femtosecond laser ablation of steel and titanium alloys," Applied Physics B, vol. 116, no. 2, pp. 485-491, 2014.
[23] J. P. Singh and S. N. Thakur, Laser-Induced Breakdown Spectroscopy, Elsevier, Amsterdam, London, 2007.

[24] K. L. Eland, D. N. Stratis, T. Lai, M. A. Berg, S. R. Goode, and S. M. Angel, "Some comparisons of LIBS measurements using nanosecond and picosecond laser pulses," Applied Spectroscopy, vol. 55, no. 3, pp. 279-285, 2001.

[25] L. Yue-Hua, C. Ming, L. Xiang-Dong, C. Qing-Qiang, and Z. Ming-Wen, "The mechanism of effect of lens-to-sample distance on laser-induced plasma," Acta Physica Sinica, vol. 62, no. 2, p. 025203, 2013.

[26] R. Junjuri, S. A. Rashkovskiy, and M. K. Gundawar, "Dependence of radiation decay constant of laser produced copper plasma on focal position," Physics of Plasmas, vol. 26, no. 12, article 122107, 2019.

[27] S. S. Mao, X. Mao, R. Greif, and R. E. Russo, "Dynamics of an air breakdown plasma on a solid surface during picosecond laser ablation," Applied Physics Letters, vol. 76, no. 1, pp. 3133, 2000.

[28] J. A. Aguilera, C. Aragón, and F. Peñalba, "Plasma shielding effect in laser ablation of metallic samples and its influence on LIBS analysis," Applied Surface Science, vol. 127-129, pp. 309-314, 1998.

[29] J. Wang, X. Li, H. Li, X. Li, and Z. Li, "Lens-to-sample distance effect on the quantitative analysis of steel by laser-induced breakdown spectroscopy," Journal of Physics D: Applied Physics, vol. 53, no. 25, article 255203, 2020.

[30] X. Li, W. Wei, J. Wu, S. Jia, and A. Qiu, "The influence of spot size on the expansion dynamics of nanosecond-laser-produced copper plasmas in atmosphere," Journal of Applied Physics, vol. 113, no. 24, article 243304, 2013.

[31] O. A. Ranjbar, Z. Lin, and A. N. Volkov, "Effect of the spot size on ionization and degree of plasma shielding in plumes induced by irradiation of a copper target by multiple short laser pulses," Applied Physics A, vol. 126, no. 5, p. 95, 2020.

[32] A. M. El Sherbini, H. Hegazy, and T. M. El Sherbini, "Measurement of electron density utilizing the $\mathrm{H}_{\alpha}$-line from laser produced plasma in air," Spectrochimica Acta Part B: Atomic Spectroscopy, vol. 61, no. 5, pp. 532-539, 2006.

[33] P. Kepple and H. R. Griem, "Improved stark profile calculations for the hydrogen lines $\mathrm{H} \alpha, \mathrm{H} \beta, \mathrm{H} \gamma$, and $\mathrm{H} \delta$," Physical Review, vol. 173, no. 1, pp. 317-325, 1968.

[34] G. Cristoforetti, A. de Giacomo, M. Dell'Aglio et al., "Local thermodynamic equilibrium in laser-induced breakdown spectroscopy: beyond the McWhirter criterion," Spectrochimica Acta Part B: Atomic Spectroscopy, vol. 65, no. 1, pp. 8695, 2010.

[35] E. Mal, R. Junjuri, M. K. Gundawar, and A. Khare, “Optimization of temporal window for application of calibration freelaser induced breakdown spectroscopy (CF-LIBS) on copper alloys in air employing a single line," Journal of Analytical Atomic Spectrometry, vol. 34, no. 2, pp. 319-330, 2019.

[36] J. Hou, L. Zhang, W. Yin et al., "Development and performance evaluation of self-absorption-free laser-induced breakdown spectroscopy for directly capturing optically thin spectral line and realizing accurate chemical composition measurements," Optics Express, vol. 25, no. 19, pp. 23024-23034, 2017.

[37] Q. Wang, A. Chen, W. Xu et al., "Effect of lens focusing distance on AlO molecular emission from femtosecond laserinduced aluminum plasma in air," Optics \& Laser Technology, vol. 122, article 105862, 2020. 